\title{
Plastic bronchitis in a 3-year-old boy
}

\author{
Xianan Zhang MD, Angela Vinturache MD PhD, Guodong Ding MD PhD
}

Cite as: CMAJ 2019 December 2;191:E1336. doi: 10.1503/cmaj.190795
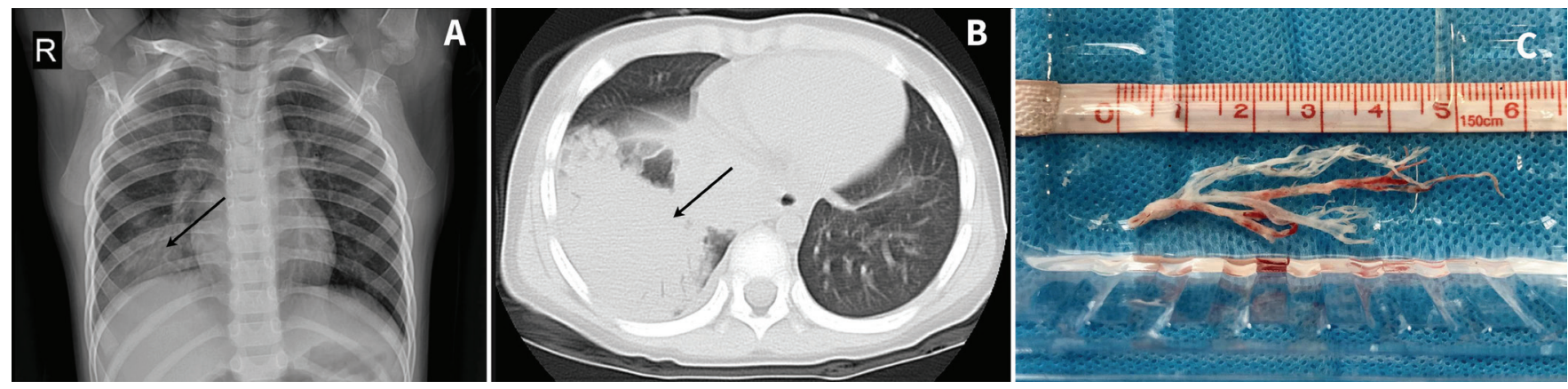

Figure 1: (A) Chest radiograph of a 3-year-old boy on the day of admission, showing right lower lobe segmental consolidation (black arrow). (B) Computed tomography scan of the patient's chest on the day of admission, showing right lower lobe consolidation and atelectasis (black arrow). (C) Cast extracted from the lumen of the right lower lobe bronchus during bronchoscopy, showing preservation of the anatomy of the bronchial tree.

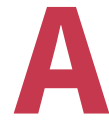

previously healthy 3-year-old boy presented to the department of respiratory medicine with a 7-day history of persistent high fever, violent cough and worsening dyspnea. Despite therapy with antibiotics and mucolytics (orally administered cefaclor [30 mg/kg/d, 3 times daily] and ambroxol hydrochloride [3 mg/ $/ \mathrm{kg} / \mathrm{d}$, twice daily] for 6 consecutive days) at the local primary health care facility, the child's respiratory status progressively deteriorated and he was referred to our institution. On presentation, the patient was in respiratory distress, with laboured breathing and decreased breath sounds in his right chest. A radiograph of his chest showed segmental consolidation in the lower lobe of the right lung (Figure 1A). The atypical presentation, age of the patient, poor response to conventional therapy and the consolidation in his right lung led us to suspect airway obstruction by foreign body, prompting further investigations. A computed tomography (CT) scan of the patient's chest showed right lower lobe atelectasis (Figure 1B). Flexible bronchoscopy showed normal anatomy, thick secretions and obstruction of the lumen of the right lower lobe bronchus with a whitish, rubbery material, consistent with an endobronchial cast. The cast was removed using forceps and aspiration (Figure 1C). Colloidal gold immunochromatographic assay of nasopharyngeal swab was positive for influenza B virus. A quantitative reverse transcription polymerase chain reaction test of the bronchoalveolar lavage fluid identified $5.8 \times 10^{3} \mathrm{DNA}$ copies $/ \mathrm{mL}$ of $\mathrm{Myco}-$ plasma pneumoniae. Bronchial cast histopathology showed an inflammatory fibrinous exudate (Appendix 1, available at www. cmaj.ca/lookup/suppl/doi:10.1503/cmaj.190795/-/DC1). Intravenous fluids, oseltamivir, azithromycin and mucolytics stabilized and improved the patient's respiratory status and he was discharged on day 12 with complete resolution of atelectasis.
Plastic bronchitis is an uncommon condition characterized by the formation of extensive obstructive airway plugs. ${ }^{1}$ Two types of the endobronchial casts have been described: ${ }^{1}$ type 1 , inflammatory casts composed of fibrin and dense inflammatory infiltrate, associated with asthma or respiratory infection; ${ }^{2}$ and type 2, acellular casts composed mostly of mucin, associated with congenital heart diseases. ${ }^{2}$ The symptomatology of plastic bronchitis is variable, from mild to life-threatening disease. The aim of treatment is to remove the casts and address the symptoms. ${ }^{3}$ This case shows that plastic bronchitis may be triggered by common respiratory tract infections and should not be overlooked in otherwise healthy children who develop atelectasis.

\section{References}

1. Brogan TV, Finn LS, Pyskaty DJ Jr, et al. Plastic bronchitis in children: a case series and review of the medical literature. Pediatr Pulmonol 2002;34:482-7.

2. Madsen P, Shah SA, Rubin BK. Plastic bronchitis: new insights and a classification scheme. Paediatr Respir Rev 2005;6:292-300.

3. Eberlein MH, Drummond MB, Haponik EF. Plastic bronchitis: a management challenge. Am J Med Sci 2008;335:163-9.

Competing interests: None declared.

This article has been peer reviewed.

The authors have obtained consent from the patient's parents.

Affiliations: Department of Respiratory Medicine (Zhang, Ding), Shanghai Children's Hospital, Shanghai Jiao Tong University, Shanghai, China; Department Obstetrics \& Gynecology (Vinturache), John Radcliffe Hospital, Oxford University Hospitals NHS Foundation Trust, Oxford, UK

Correspondence to: Guodong Ding, dingguodong@shchildren.com.cn 